\title{
The effects of compression garments and electrostimulation on athletes' muscle soreness and recovery
}

\author{
Yunus Turgay Erten', Turker Sahinkaya', Engin Dinc', Bekir Eray Kilinc ${ }^{2, *}$, Bulent Bayraktar', Mehmet Kurtoglu' \\ 'Sports Medicine, Istanbul Medical School, Istanbul, Turkey \\ ${ }^{2}$ Orthopaedics, Igdir State Hospital, Igdir, Turkey
}

In this study, we explained the effects of compression garment and electrostimulation on athletes' recovery period by evaluating blood lactate and isokinetic peak torque parameters. Twenty volunteers (15.55 \pm $0.51 \mathrm{yr}$ ) were included to study. At recovery period, blood samples was taken for lactate values at 0th, 3rd, 5th, 15th, 30th min. The isokinetic strength test was performed on right ankle at 15th min and on the left ankle at 30th min. The same protocol was performed for compression garment on 2 weeks and for electrostimulation on third weeks and results were compared. There wasn't any significant difference on blood lactate levels within groups. At women; there was not any significant difference on isokinetic peak torques within two groups. but at electrostimulation usage we found significant increases on right plantar flexion $(P<0.1)$, right dorsal flexion (RDF) $(P<0.1)$ and left plantar flexion (LPF) $(P<0.1)$ values compared to control measurements. At men; with com- pression garment usage, there was significant increase on LPF values compared to control measurements. At electrostimulation usage, we found significant increases on $\operatorname{RDF}(P<0.1)$ and left dorsal flexion $(P<0.1)$ values compared to control measurements. During recovery, there is not any beneficial effect seen on blood lactate level within two groups. When compared to passive rest, compression garments and electrostimulation interventions effects on force generation capacity at recovery are statically significant. Also in terms of force generation capacity; usage of electrostimulation during $15 \mathrm{~min}$ and compression garments during 30 min were statically more significant.

Keywords: Compression garments, Electrostimulation, Muscle soreness, Recovery period of exercise

\section{INTRODUCTION}

Athletes' health and sports performance are very important issues that have been researched nowadays. When think about individiually, it is easily understood that athletes injury prevention and sports performance development is very crucial. Researches about effective recovery methods have been doing recently.

Performance in sport is closely related with lactate metabolism and level in the blood (Karlsson et.al., 1975; Klausen et al., 1972). Elimination of the lactate in the blood is the most effective way to increase the muscle performance and strenght (Stamford et al., 1978).

In our study; our aim was to explain the effects of compression

${ }^{*}$ Corresponding author: Bekir Eray Kilinc (D) http://orcid.org/0000-0003-1229-9815 Orthopaedics, Igdir State Hospital, Mehmet Akif Mah.23 Nisan Cad.No:66/2 Bucak/Burdur 15300, Turkey

Tel: +90-530-606-1884, Fax: +90-248-325-2161, E-mail: dreraykilinc@gmail.com Received: May 20, 2016 / Accepted: July 23, 2016 garment and electrostimulation on athletes' recovery period by evaluating blood lactate and isokinetic peak torque parameters. We investigated the effects of compression stocking and electrostimulation on blood lactate value and isokinetic strength value during the first 30 min of recovery after 30 min of aerobic running the treadmill at anaerobic threshold heart rate of $85 \%$.

\section{MATERIALS AND METHODS}

Nine female volleyball players (age, $15.89 \pm 0.33 \mathrm{yr}$; height, $171.78 \pm 9.52 \mathrm{~cm}$; weight, $60.44 \pm 4.97 \mathrm{~kg}$ ) and 11 male basketball players (age, $15.27 \pm 0.46 \mathrm{yr}$; height, $182.55 \pm 5.75 \mathrm{~cm}$; weight, $70.73 \pm 12.81 \mathrm{~kg}$ ) totally 20 athletes (age, $15.55 \pm 0.51$ years; 
height, $177.7 \pm 9.26 \mathrm{~cm}$; weight, $66.1 \pm 11.15 \mathrm{~kg}$ ) were included the study. The athletes were training at least $3 \mathrm{yr}$ and $8 \mathrm{hr}$ a week. Inclusion criteria included normal venous capacity and no arterial disorder. None of the participants had a history of heart or circulation disorder. Before the study, each participant was informed about the purpose and risk of the study and signed an informed consent.

\section{Preliminary testing}

Methodologically, athletes were conducted two different laboratory test in the first week of laboratory visit. The first test was cardiopulmonar exercise test. It was performed for the determination of maximal oxygen consumption $\left(\mathrm{VO}_{2 \max }, \mathrm{mL} / \mathrm{kg}\right.$. $\mathrm{min}$ ) and anaerobic threshold pulse. The second test was isokinetic muscle test. It is performed for determination of the triceps surae and tibialis anterior physiologic power and endurance parameters. There were at least $48 \mathrm{hr}$ between two test protocol.

The athletes had breakfast $2 \mathrm{hr}$ before laboratory visit. Firstly all athletes were taken detailed medical history. The heights and weights of the athletes were evaluated. During systemical physical examination, there were not any contraindication identified for cardiopulmonar exercise testing. Firstly resting heart rate and resting blood pressure was measured and recorded. Then, the athletes were conducted specific mask (Metalyzer 3B, Cortex, Leipzig, Germany) for determination of anaerobic threshold pulse and $\mathrm{VO}_{2 \text { max }}$ by evaluating $\mathrm{CO}_{2}$ and $\mathrm{O}_{2}$ levels (Metasoft 3, Cortex) via $\mathrm{V}$ slope method. During cardiopulmonar exercise testing, all participants were monitored and electrocardiography changes and blood pressure were recorded every $3 \mathrm{~min}$. The participants were run until personal exhaustion happened unless identification of any symptom or sign required to stop Bruce test protocol. In our study, during cardiopulmonar exercise test, the last 10-sec maximal average value on the peak exercise was determined $\mathrm{VO}_{2}$ max. Anaerobic threshold pulses were determined personally via $\mathrm{V}$ slope method. Eight-five percent of anaerobic threshold pulse was identified personally in order to make athletes exercise on the aerobic metabolism for the next experimental test protocols.

The isokinetic dynamometer test was planned as a second test for determining triceps surae and tibialis anterior physiologic power and endurance parameters. Before isokinetic testing, participiants cycled $10 \mathrm{~min}$ on 50-60 rpm for warming on the horizantal exercise cycle (Bike 5150R, SportsArt, Tainan, Taiwan). After warming period, the participants performed low extremity stretching exercises for $5 \mathrm{~min}$. Immediately after stretching period, the isokinetic test (Cybex Extremity System) was performed for right and left ankle. The right and left triceps suare and tibialis anterior peak torque was evaluated on the $30 \%$ sec, endurance was evaluated on the $120 \%$ sec and physiological datas were recorded.

\section{Compression stockings}

Sigvaris compression stocking (poliamid \%64, polyester \%17, elastane \%19; Ganzoni \& Cie AG, Galen, Switzerland) was used (20-30 mmHg).

\section{Electrostimulation device}

Veinoplus Neuromuscular Stimulator was used for electrostimulation (low-frequency electrostimulation) (Ad Rem Technology, rue de Faubourg Saint Honore, Paris, France).

Experiment protocol was planned in three different sessions. The first session was designed as a control session. In the second session, experiment protocol was performed with the compression stocking. In the third session, experiment protocol was performed with the usage of electrostimulation device (low-frequency electrostimulation). Isokinetic test results for both ankle were planned as performance indicators during 15 th $\mathrm{min}$. and at the end of recovery period. During three sessions, the first isokinetic test was planned on the 15th min of the recovery period because the half time of the most team sport and individual sport lasts $15 \mathrm{~min}$. The second isokinetic test was planned on the 30th min of the recovery period (Fig. 1).

\section{Experımental desıgn}

Athletes were exercised on treadmill on the pulse of $85 \%$ anaerobic threshold threshold value during $30 \mathrm{~min}$ in order to provide aerobic exhaustion. During exercise, athletes pulse value was

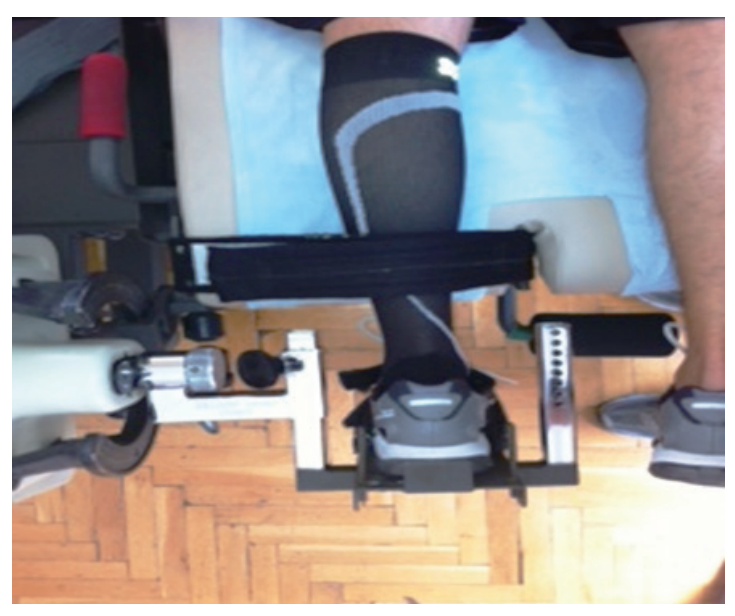

Fig. 1. Isokinetic test. 
monitored. After exercise period, athletes were sat on a chair for 30th min recovery period. During recovery period, blood samples were taken on the 0 th, 3rd, 5th, 15th, 30th min from athletes' finger and this blood samples were evaluated and results were recorded. First isokinetic test was performed to the right ankle of the athletes during the 15th min of recovery period and numeric datas were recorded. Second isokinetic test was performed to the left ankle of the athletes during the 30th min of recovery period and numeric datas were recorded.

Same protocol was applied for athletes before exercise period. After exercise period, compression stockings were worn before athletes were sat on a chair for 30th min recovery period, and same protocol applied after wearing compression stockings.

Same protocol was applied for athletes before exercise period. During recovery period, electrostimulation was applied on to the bilateral calf muscles, same protocol applied after applying electrostimulation.

The blood lactate values and isokinetic parameters during sessions, two tests were used to statistical analysis. Wilcoxon signed rank test was used to compare two dependent variables. Mann Whitney $U$-test was used to compare two independent variables.

Table 1. Blood lactat values (mmol/L)

\begin{tabular}{lccc}
\hline Recovery period $(\mathrm{min})$ & Control & Garment & Electrositimulation \\
\hline Oth & $3.08 \pm 1.19$ & $2.70 \pm 1.03$ & $3.37 \pm 1.47$ \\
3th & $2.10 \pm 0.49$ & $3.12 \pm 1.19$ & $2.33 \pm 0.75$ \\
5th & $1.83 \pm 0.43$ & $2.68 \pm 0.89$ & $1.87 \pm 0.43$ \\
15th & $2.37 \pm 1.88$ & $2.11 \pm 0.42$ & $1.94 \pm 0.47$ \\
30th & $2.07 \pm 0.60$ & $2.39 \pm 0.67$ & $2.59 \pm 0.76$
\end{tabular}

Values are presented as mean \pm standard deviation.

Table 2. Comparison control group with garment group in isokinetic power $(\mathrm{n}=20)$

\begin{tabular}{llll}
\hline Voluntary contraction & Control & Garment & $P$-value \\
\hline RPF & & & \\
Peak & 120 & 127.3 & 0.017 \\
\%BW & 181.3 & 192.65 & 0.018 \\
RDF & & & \\
Peak & 40.5 & 41.6 & 0.265 \\
\%BW & 61.15 & 63.7 & 0.184 \\
LPF & & & \\
Peak & 122 & 128.3 & 0.009 \\
\%BW & 184.55 & 194.75 & 0.01 \\
LDF & & & \\
Peak & 41.9 & 42.65 & 0.583 \\
\%BW & 63.65 & 65.7 & 0.216 \\
\hline
\end{tabular}

$\mathrm{RPF}$, right plantar flexion maximal voluntary contraction; RDF, right dorsal flexion maximal voluntary contraction; LPF, left plantar flexion maximal voluntary contraction; LDF, left dorsal flexion maximal voluntary contraction; BW, body weight.
The value of $P<0.1$ for all analysis was accepted statistically significant.

\section{RESULTS}

There was no significant difference on blood lactate levels within measurements (Table I). At women; there was no significant difference on isokinetic peak torques within compression garment measurements and control measurements. At electrostimulation measurements there was a significant increases on right plantar flexion (RPF) $(P<0.01)$, right dorsal flexion (RDF) $(P<0.1)$, and left plantar flexion (LPF) $(P<0.1)$ values compared to control measurements.

At men, with compression garment measurements, there was a significant increase on LPF values compared to control measurements $(P<0.1)$. At electrostimulation measurements, there was a significant increases on RPF $(P<0.1)$ and LPF $(P<0.1)$ values compared to control measurements.

Compression stocking and electrostimulation intervention effects on force generating capacity on isokinetic device during recovery period had statistically significant compared to passive recovery. In terms of the force generating capacity, electrostimulation intervention at 15 th min of recovery and wearing compression stocking at the 30th min of recovery were more statistically significant (Tables 2, 3).

\section{DISCUSSION}

It was shown that there is a close relationship between lactate

Table 3. Comparison control group with electrostimulation group in isokinetic power $(n=20)$

\begin{tabular}{lllr}
\hline Voluntary contraction & Control & Garment & $P$-value \\
\hline $\begin{array}{lllr}\text { RPF } \\
\text { Peak }\end{array}$ & 120 & 135.65 & 0.001 \\
\%BW & 181.3 & 206.7 & $<0.001$ \\
RDF & & & \\
Peak & 40.5 & 42.85 & 0.016 \\
\%BW & 61.15 & 65.35 & 0.004 \\
LPF & & & \\
Peak & 122 & 136.65 & 0.001 \\
\%BW & 184.55 & 208.2 & 0.001 \\
LDF & & & \\
Peak & 41.9 & 43.05 & 0.367 \\
\%BW & 63.65 & 65.35 & 0.391 \\
\hline
\end{tabular}

RPF, right plantar flexion maximal voluntary contraction; RDF, right dorsal flexion maximal voluntary contraction; LPF, left plantar flexion maximal voluntary contraction; LDF, left dorsal flexion maximal voluntary contraction; BW, body weight. 
metabolism and exercise performance; work capacity and performance are affected negatively by increased blood lactate level (Bangsbo et al., 1996; Klausen et al., 1972; Stamford et al., 1978). The impact of the various recovery protocols on blood lactate elimination following maximal exercise was investigated. Active recovery process was showed to be the most effective way in ensuring the elimination of lactate. To be sure, assuming that the active recovery has contributed to the increase of working muscles metabolism and blood flow in aerobic environment, the active recovery is believed to result in lactate distribution and transformation as a substrate for oxidation (Bangsbo et al., 1994; Brooks, 1986; Hatta, 1990; Martin et al., 1998; Monedero and Donne 2000). In our study; it was thought that the lactate value differences between 0th min control measurement belonging to same female athletes and measurements of compression stocking and electrostimulation application could depend on sleep and rest durations of the previous night, eating habits, rest status for the last $24 \mathrm{hr}$ and nourishment situation during that day of the athlete. In the application of control and electrostimulation during the process between the 0th and the 5 th min of the recovery, blood lactate level, while tending to fall, were detected to increase a little amount when compression stocking is worn. This increase might be due to the difficulty and stress during wearing of compression stocking. In the period from the 5th to 15 th min of the recovery, it was observed that blood lactate values obtained from compression stockings and electrostimulation applications are in a downward tendency while blood lactate values in control application are in an upward tendency. This change is not statistically significant. In the 15th min of the recovery, while the lowest blood lactate values are observed in the measurements to which the electrostimulation applied, the highest values are seen in control measurements.

According to the control measurements; this decrease observed on the people who are wearing compression stockings and to whom electrostimulation is applied is not statistically significant. Blood lactate values in the people wearing compression stockings were found to be higher than those to whom electrostimulation is applied. Between the 15th and the 30th min of the recovery period, while blood lactate values of the subjects wearing compression stocking and undergoing electrostimulation application tend to increase, blood lactate values of the subjects in control group tend to decrease.

When blood lactate levels of male and female athletes are evaluated together, the highest blood lactate values are observed in control measurements in the 15 th min of the recovery while the lower lactate values are observed in the athletes wearing compression stockings. The lowest blood lactate values are observed in electrostimulation application. These differences are not statistically significant. After $15 \mathrm{~min}$, while the blood lactate values tend to decrease in control measurements; they tend to increase in those wearing compression stockings and undergoing electrostimulation application.

To evaluate the effects of compression stockings on the changes of athletic performance indication, lower lactate and haematocrit levels were detected after exercise in those wearing compression stockings (Berry and McMurray, 1987; Chatard et al., 2004). In our study, in the 15 th min of the recovery after $30 \mathrm{~min}$ of aerobic exercise, blood lactate values are observed to be lower in females and total compression stockings implementation than those observed in control group, but the difference was not statistically significant.

Among many possible effective recovery modalities, athletes also use electrostimulation application as well. There are limited studies and scientific evidence related to the effect of many physiological variables on the increase of recovery kinetic, continuance of athletic performance and the decrease of rating of perceived exertion. In the studies comparing the different postexercise recovery periods, no significant difference in lactate levels were determined (Cortis et al., 2010; Tessitore et al., 2008). Active recovery and electrostimulation application were observed to have longer climbing periods and more positive effects on blood lactate concentrations than passive recovery (Heyman et al., 2009; Neric et al., 2009). In our study, we found that the highest lactate values in the 15th min of recovery period after 30th min of aerobic exercise is in control measurements at rest sitting. Even if blood lactate levels were determined to be lower in those wearing compression stockings and higher in those experiencing electrostimulation application, these differences were statistically significant. We used isokinetic strength values as a second parameter in researching the effects of compression stockings electrostimulation application on the recovery. In the 15 th min of the recovery in electrostimulation application of females, we determined the significant peak RPF increase in comparison with control measurements. Likewise, in the 15th min of recovery, we determined the statistically significant increase in the peak RDF. We did not determine significant changes in the peak RPF and peak RDF values arising from compression stockings implementation for both parameters as well. In the peak LPF values evaluated in the 30th min of recovery, we determined significant increase compared to the control measurements. In the peak LPF values of compression stock- 
ings application, we did not find a significant change compared to control measurements. According to the peak LDF values evaluated in the 30th min of recovery; we did not identify significant changes in compression stockings and electrostimulation applications. In the peak RPF values evaluated in the 15th min of recovery in males, we identified a significant increase in electrostimulation applications compared to control measurements. We did not identify a significant change in compression stockings application compared to control measurements. In the peak LPF values evaluated in the 30th min of recovery, we identified significant increases both in compression stockings and in electrostimulation applications compared to control measurements. In the peak LDF values, another parameter evaluated in the 30th min of recovery, we did not identify significant changes neither in stockings nor in electrostimulation applications.

Compression stockings used before exercise, during exercise and after exercise were showed not to have an effect on blood lactate levels and not to have developed the recovery strength in the first 30 min (Duffield et al., 2008; Duffield et al., 2010; Maton et al., 2006). In the studies evaluating the effects of the use of compression stockings on muscle fatigue and muscle concentric strength, compression stockings were found to alleviate the decrease in concentric strength and muscle fatigue compared to control group (Ali et al., 2007; Duffield and Portus, 2007; Jakeman et al., 2010; Perrey et al., 2008). In our study, we used compression stockings with a pressure capacity of $30 \mathrm{mmHg}$ at the level of ankle, and compression stockings with a pressure capacity of $20 \mathrm{mmHg}$ whose intensity gradually decreases up to the knee, even if we did not observed a significant strength increase in females compared to control group, we found a significant increase in LPF strength parameters of males. In the peak strength values of RPF, RDF, and LDF which are other strength parameters, although increases were found in the group wearing compression stockings in comparison with control groups, there was not a statistically significant difference.

In recovery process; passive recovery, active recovery (running on $50 \%$ of aerobic capacity) and the effect of low-frequency electrostimulation were compared with each other. When knee extensor contractions with maximal voluntary were compared, it was stated that electrostimulation approach leads to a better contraction intensity, not showing a significant difference though, on the other hand, there is a need for further studies on this issue (Cortis et al., 2010; Grunovas et al., 2007; Lattier et al., 2004; McLoughlin et al., 2004; Tessitore et al., 2007; Vanderthommen et al., 2007). In our study, with low-frequency electrostimulation during 30th min of recovery process after 30th min of aerobic exercise, we compared the control measurements in terms of isokinetic values for right ankle in the 15th min. We identified significant increases in RPF, RDF, and LPF isokinetic strength values in females undergoing electrostimulation compared to control measurements. As for males undergoing electrostimulation, we identified significant increases in RPL and LPL isokinetic strength values compared to control measurements. When we evaluate the results of the study that we conducted and compare this with the other studies; we consider that electrostimulation application can be an alternative method in recovery period.

During recovery, when compared to control measurement, there is not any beneficial effect seen on blood lactate level within compression garment measurements and electrostimulation measurements. When compared to passive rest, compression garments and electrostimulation interventions effects on force generation capacity at recovery are statically significant. If we consider the low level of lactate at the 15 th min of recovery period especially on electrostimulation group, applying electrostimulation during halftime of activities like football, basketball seems to be beneficial. There has to be more comprehensive studies needed among bigger populations to evaluate the effectiveness of compression garments and electrostimulation on athletes recovery period.

\section{CONFLICT OF INTEREST}

No potential conflict of interest relevant to this article was reported.

\section{REFERENCES}

Ali A, Caine MP, Snow BG. Graduated compression stockings: physiological and perceptual responses during and after exercise. J Sports Sci 2007;25:413-419.

Bangsbo J, Graham T, Johansen L, Saltin B. Muscle lactate metabolism in recovery from intense exhaustive exercise: impact of light exercise. J Appl Physiol (1985) 1994;77:1890-1895.

Bangsbo J, Madsen K, Kiens B, Richter EA. Effect of muscle acidity on muscle metabolism and fatigue during intense exercise in man. J Physiol 1996;495 (Pt 2):587-596.

Berry MJ, McMurray RG. Effects of graduated compression stockings on blood lactate following an exhaustive bout of exercise. Am J Phys Med 1987;66:121-132.

Brooks GA. The lactate shuttle during exercise and recovery. Med Sci Sports Exerc 1986;18:360-368. 
Chatard JC, Atlaoui D, Farjanel J, Louisy F, Rastel D, Guézennec CY. Elastic stockings, performance and leg pain recovery in 63-year-old sportsmen. Eur J Appl Physiol 2004;93:347-352.

Cortis C, Tessitore A, D'Artibale E, Meeusen R, Capranica L. Effects of post-exercise recovery interventions on physiological, psychological, and performance parameters. Int J Sports Med 2010;31:327-335.

Duffield R, Cannon J, King M. The effects of compression garments on recovery of muscle performance following high-intensity sprint and plyometric exercise. J Sci Med Sport 2010;13:136-140.

Duffield R, Edge J, Merrells R, Hawke E, Barnes M, Simcock D, Gill N. The effects of compression garments on intermittent exercise performance and recovery on consecutive days. Int J Sports Physiol Perform 2008;3:454-468.

Duffield R, Portus M. Comparison of three types of full-body compression garments on throwing and repeat-sprint performance in cricket players. Br J Sports Med 2007;41:409-414.

Grunovas A, Silinskas V, Poderys J, Trinkunas E. Peripheral and systemic circulation after local dynamic exercise and recovery using passive foot movement and electrostimulation. J Sports Med Phys Fitness 2007;47:335-343.

Hatta H. Oxidative removal of lactate after strenuous exercise. Ann Physiol Anthropol 1990;9:213-218.

Heyman E, DE Geus B, Mertens I, Meeusen R. Effects of four recovery methods on repeated maximal rock climbing performance. Med Sci Sports Exerc 2009;41:1303-1310.

Jakeman JR, Byrne C, Eston RG. Lower limb compression garment improves recovery from exercise-induced muscle damage in young, active females. Eur J Appl Physiol 2010;109:1137-1144.

Karlsson J, Bonde-Petersen F, Henriksson J, Knuttgen HG. Effects of previous exercise with arms or legs on metabolism and performance in exhaustive exercise. J Appl Physiol 1975;38:763-767.

Klausen K, Knuttgen HG, Forster HV. Effect of pre-existing high blood lactate concentration on maximal exercise performance. Scand J Clin Lab Invest 1972;30:415-419.
Lattier G, Millet GY, Martin A, Martin V. Fatigue and recovery after high-intensity exercise. Part II: Recovery interventions. Int J Sports Med 2004;25:509-515.

Martin NA, Zoeller RF, Robertson RJ, Lephart SM. The comparative effects of sports massage, active recovery, and rest in promoting blood lactate clearance after supramaximal leg exercise. J Athl Train 1998; 33:30-35.

Maton B, Thiney G, Dang S, Tra S, Bassez S, Wicart P, Ouchene A. Human muscle fatigue and elastic compressive stockings. Eur J Appl Physiol 2006;97:432-442.

McLoughlin TJ, Snyder AR, Brolinson PG, Pizza FX. Sensory level electrical muscle stimulation: effect on markers of muscle injury. Br J Sports Med 2004;38:725-729.

Monedero J, Donne B. Effect of recovery interventions on lactate removal and subsequent performance. Int J Sports Med 2000;21:593-597.

Neric FB, Beam WC, Brown LE, Wiersma LD. Comparison of swim recovery and muscle stimulation on lactate removal after sprint swimming. J Strength Cond Res 2009;23:2560-2567.

Perrey S, Bringard A, Racinais S, Puchaux K, Belluye N, Estivalet M, Brisson P. Graduated compression stockings and delayed onset muscle soreness (P105). In: Estivalet M, Brisson P, editors. The engineering of sport 7. Paris: Springer; 2008. p. 547-554.

Stamford BA, Moffatt RJ, Weltman A, Maldonado C, Curtis M. Blood lactate disappearance after supramaximal one-legged exercise. J Appl Physiol Respir Environ Exerc Physiol 1978;45:244-248.

Tessitore A, Meeusen R, Cortis C, Capranica L. Effects of different recovery interventions on anaerobic performances following preseason soccer training. J Strength Cond Res 2007;21:745-750.

Tessitore A, Meeusen R, Pagano R, Benvenuti C, Tiberi M, Capranica L. Effectiveness of active versus passive recovery strategies after futsal games. J Strength Cond Res 2008;22:1402-1412.

Vanderthommen M, Soltani K, Maquet D, Crielaard JM, Croisier JL. Does neuromuscular electrical stimulation influence muscle recovery after maximal isokinetic exercise? Isokinet Exerc Sci 2007;15:143-149. 